\title{
Growth of a Diffusion Flame in the Field of a Vortex
}

\author{
F. E. MARble
}

ABSTRACT. A simple diffusion flame with fast chemical kinetics is initiated along the horizontal axis between a fuel occupying the upper half-plane and an oxidizer below. Simultaneously a vortex of circulation $\Gamma$ is established at the origin. As time progresses the flame is extended and "wound up" by the vortex flow field and the viscous core of the vortex spreads, converting the motion in the core to a solid-body rotation.

The kinematics of the flame extension and distortion is described and the effect of the local-flow field upon local-flame structure is. analyzed in detail. It is shown that the combustion field consists of a totally reacted core region, whose radius is time dependent, and an external flame region consisting of a pair of spiral arms extending off at large radii toward their original positions on the horizontal axis.

The growth of the reacted core, and the reactant-consumption rate augmentation by the vortex field in both core and outer-flame regions were determined for values of the Reynolds number $(\Gamma / 2 \pi \nu)$ between 1 and $10^{3}$ and for a wide range of Schmidt numbers $(\nu / D)$ covering both gas and liquid reactions.

For large values of Reynolds number the radius $r_{*}$ of the reactant grows much more rapidly than the viscous core so that only the nearly inviscid portion of the flow is involved. The more accurate condition for this behavior is that $R(\mathrm{Sc})^{1 / 2}>50$ and, under this restriction, the similarity rule for the core radius growth is shown to be

$$
\frac{r_{*}}{\left(\Gamma^{2 / 3} D^{1 / 3} t\right)^{1 / 2}} \equiv 0.5092+O(D / \Gamma)^{1 / 2}
$$

In this case also the reactant consumption rate becomes independent of time and, for the complete diffusion flame in the vortex field, the augmentation of reactant-consumption rate due to the vortex field

F. E. MARBLE - California Institute of Technology, 205-45, Pasadena, CA 91125, U.S.A. 
satisfies

$\frac{\text { Augmented consumption rate }}{\Gamma^{2 / 3} D^{1 / 3}} \equiv 1.2327-1.4527(D / \Gamma)^{1 / 6}+O(D / \Gamma)^{1 / 2}$

Both of these similarity rules are, as is appropriate for high Reynolds number, independent of kinematic viscosity.

\section{INTRODUCTION}

The development of the theory of flames in nonuniform flow fields has been limited by the complications introduced into the flame structure itself as well as the rather strong effect of sensible heat release on the flow field. The diffusion flame has a relative simplicity that allows more latitude in such an analysis particularly when the chemistry is fast and the diffusion is the only rate-controlling process. In this way it has been possible to examine diffusion flames under conditions of straining in the plane of the flame,$^{(1)}$ a condition that occurs in the immediate vicinity of a stagnation point ${ }^{(2)}$ and describes locally some aspects of turbulent diffusion flames. ${ }^{(3)}$

The analysis of combustion-flow fields in the large is a much more challenging problem and the difficulty is amplified when the flow field and flame structure are nonstationary. The distortion of a diffusion flame by a viscous vortex flow, the problem considered here, has features that make it particularly significant. First, the vortex field provides a strong, variable, time-dependent strain rate that can still be handled analytically. Second, the example provides an excellent opportunity to study the behavior of diffusion flames in a viscous-dominated region of the flow, a situation that may be of significance in turbulent diffusion flames. Finally, this particular chemically reacting flow field occurs naturally in the large-scale structures of turbulent diffusion flames and in combustion-instability problems.

\section{KINEMATICS OF THE DIFFUSION FLAME-VORTEX INTERACTION}

Suppose that initially the upper and lower half-planes consist of a fuel and oxidizer respectively, that the chemical kinetics are sufficiently fast, that the reaction is diffusion controlled, and that the stoichiometry of the reaction is unity, i.e., the mass consumption of the two reactants is equal. In the absence of any fluid motion, this situation would lead to a diffusion 
flame located on the horizontal axis. The effective thickness of the diffusion zones would behave as $(D t)^{1 / 2}$ while the reactant consumption rate would vary as approximately $(D / t)^{1 / 2}$, where $D$ is the common binary diffusion coefficient of either reactant in the product. In the following analysis we shall neglect the variation of density resulting from the release of chemical energy by the reaction; it will be indicated later that this has little effect upon the results.

Now if, at $t=0$, we situate a point vortex at the origin, the inducedflow field is given by the tangential velocity ${ }^{(4)}$

$$
v_{\theta} \equiv r \frac{\partial \theta}{\partial t}=-\frac{\Gamma}{2 \pi r}\left(1-e^{-\eta^{2}}\right)
$$

where $r$ and $\theta$ are the polar coordinates of an arbitrary point in the plane, $\Gamma$ is the circulation of the vortex for large radii, $\nu$ is the kinematic viscosity, and

$$
\eta=r /(4 \nu t)^{1 / 2}
$$

is the dimensionless radius. The distortion of the flame front by this flow follows by noting that any element, originally on the horizontal axis, is transported at constant radius through an angle $\theta$ obtained by integrating equation (1) at constant radius. Then if the initial position of the flame element lies to the right of the vortex position, its angle at time $t$ is

$$
\theta(r, t)=-\frac{\Gamma}{2 \pi r^{2}} \int_{0}^{t}\left[1-\exp \left(-r^{2} / 4 \nu t_{1}\right)\right] d t_{1}
$$

which has a logarithmic singularity at $r=0, t>0$. A second branch of the curve arises from points initially to the left of the vortex and moves to an angle

$$
\theta(r, t)=\pi-\frac{\Gamma}{2 \pi r^{2}} \int_{0}^{t}\left[1-\exp \left(-\eta_{1}^{2}\right)\right] d t_{1}
$$

The dimensionless group $\Gamma / 2 \pi \nu$ will be denoted the Reynolds number, $R$, of the vortex. A flame contour is shown in Figure 1 for a Reynolds number equal to 40 and at a time after starting the motion such that $(4 \nu t)^{1 / 2}=0.1$ $\mathrm{cm}$. For larger radii the displacement is essentially that corresponding to an inviscid vortex flow while for $r<1 \mathrm{~mm}$ the viscous effects are dominant and the displacements, at this particular time, correspond to those of a solid-body rotation. At earlier time, before the viscous core has spread to 


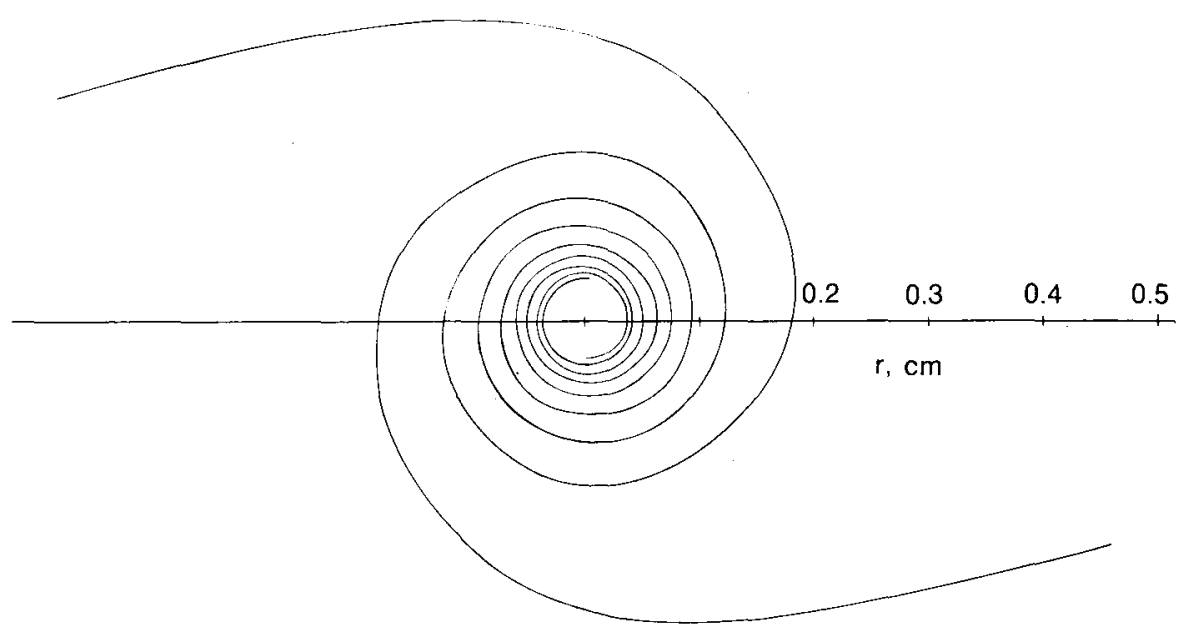

Fig. 1. Core region of distorted flame sheet, $\Gamma / 2 \pi \nu=40,(4 \nu t)^{1 / 2}=0.1 \mathrm{~cm}$.

$r \sim 0.1 \mathrm{~cm}$, a considerable degree of winding has taken place and, as the growing viscous core dominates the flow to that radius, the spiral-like distortion is "frozen" into the fluid. The contours for $r<0.1 \mathrm{~cm}$ are essentially frozen into the flow at this time. Note that the distances between neighboring flame surfaces are of the order of tenths of a millimeter. The Reynolds number determines the degree of winding that has taken place before the viscous core has spread and stopped further distortion. For values of $R$ equal to approximately 1000 , the structure is extremely fine; for $R$ about 5 , the structure is rather coarse.

To analyze the effect of the flow field on the local-flame structure it is necessary to determine the history of the flow that is observed by a flame element in its immediate neighborhood. Figure 2 shows a flame element at three successive times; the termini of the element lie on fixed circles and the increase in element length and its rotation with respect to a local tangent are established by the velocity difference between the two radii.

If this flame element lies outside the region of viscous influence, then at a radius $r$ the gas will move distance $\Gamma t / 2 \pi r$ in time $t$, and the length $d s$ of a flame element initially of length $d r$ and situated on the horizontal axis is given by

$$
d s=\left[1+\left(\frac{\Gamma t}{\pi r^{2}}\right)^{2}\right]^{1 / 2} d r \equiv\left(1+4 \zeta^{2}\right)^{1 / 2} d r
$$






Fig. 2. Schematic drawing of distortion and rotation of a flame element at successive times.

for $\zeta \gg 1$, the ratio of flame-element length to its initial length is

$$
d s / d r \cong 2 \zeta \equiv \Gamma t / \pi r^{2}
$$

This quantity will be convenient in our calculations because it clarifies the effect of Reynolds number upon an otherwise ideal-fluid result. For example, the angle $\theta$ though which a point has moved becomes, from equation (1),

$$
\theta=-\int_{0}^{\zeta}\left(1-e^{-R / 4 \zeta}\right) d \zeta
$$

The angle $\psi$ that the flame element makes with respect to the local tangential direction follows directly by differentiating equation (2) or (3) with respect to $r$ at constant $t$. Thus $r \partial \theta / \partial r=\cot \psi$ and hence

$$
\tan \psi=1 /\left[2 \zeta\left(1-e^{-R / 4 \zeta}\right)\right]
$$

At a fixed radius, as time increases, the flame angle $\psi$ decreases toward $\tan ^{-1}(2 / R)$, demonstrating the very tight winding that occurs for large 
Reynolds numbers before viscosity prevents further changes. For large radii at fixed time, $\zeta$ becomes small and $\psi \cong \pi / 2$, indicating that the interface is only slightly displaced from its initial position on the horizontal axis.

To examine the flow field in the neighborhood of the flame element, construct a coordinate system from the midpoint of the element with $x$-axis in the plane of the element and $y$-axis normal to it. To determine the $u$ and $v$ velocity components in this reference frame we must use the velocity field given by equation (1) and account for the orientation and the angular velocity $\partial \psi / \partial t$ of the flame element. After some caiculation it follows that these velocity components are given by

$$
\begin{gathered}
u=r \frac{\partial}{\partial r}\left(v_{\theta} / r\right)\left[\left(\frac{1}{2} \sin 2 \psi\right) x+(\cos 2 \psi) y\right] \\
v=-r \frac{\partial}{\partial r}\left(v_{\theta} / r\right)\left(\frac{1}{2} \sin 2 \psi\right) y
\end{gathered}
$$

where $x$ and $y$ represent distances in the $x-y$ coordinate system appropriately erected at the point $r, \theta$ in the polar-coordinate system. Equations (6) and (7) represent, in fact, the first terms in the Taylor expansion of the velocity field about $r, \theta$ and hence $x$ and $y$ must be small in comparison with distances over which significant changes take place in the velocity field. Simultaneously we may deduce that the angular velocity of the element is

$$
\partial \psi / \partial t=-r \frac{\partial}{\partial r}\left(v_{\theta} / r\right) \sin ^{2} \psi
$$

which follows from the condition that the velocity component $v$, normal to the element, must vanish on the element.

Now the local-flow field, given by equations (6) and (7), may be decomposed into two components: a normal straining motion

$$
\begin{gathered}
u_{1}=r \frac{\partial}{\partial r}\left(v_{\theta} / r\right)\left(\frac{1}{2} \sin 2 \psi\right) x \\
v_{1}=-r \frac{\partial}{\partial r}\left(v_{\theta} / r\right)\left(\frac{1}{2} \sin 2 \psi\right) y
\end{gathered}
$$

and a shearing motion

$$
u_{2}=r \frac{\partial}{\partial r}\left(v_{\theta} / r\right)(\cos 2 \psi) y
$$




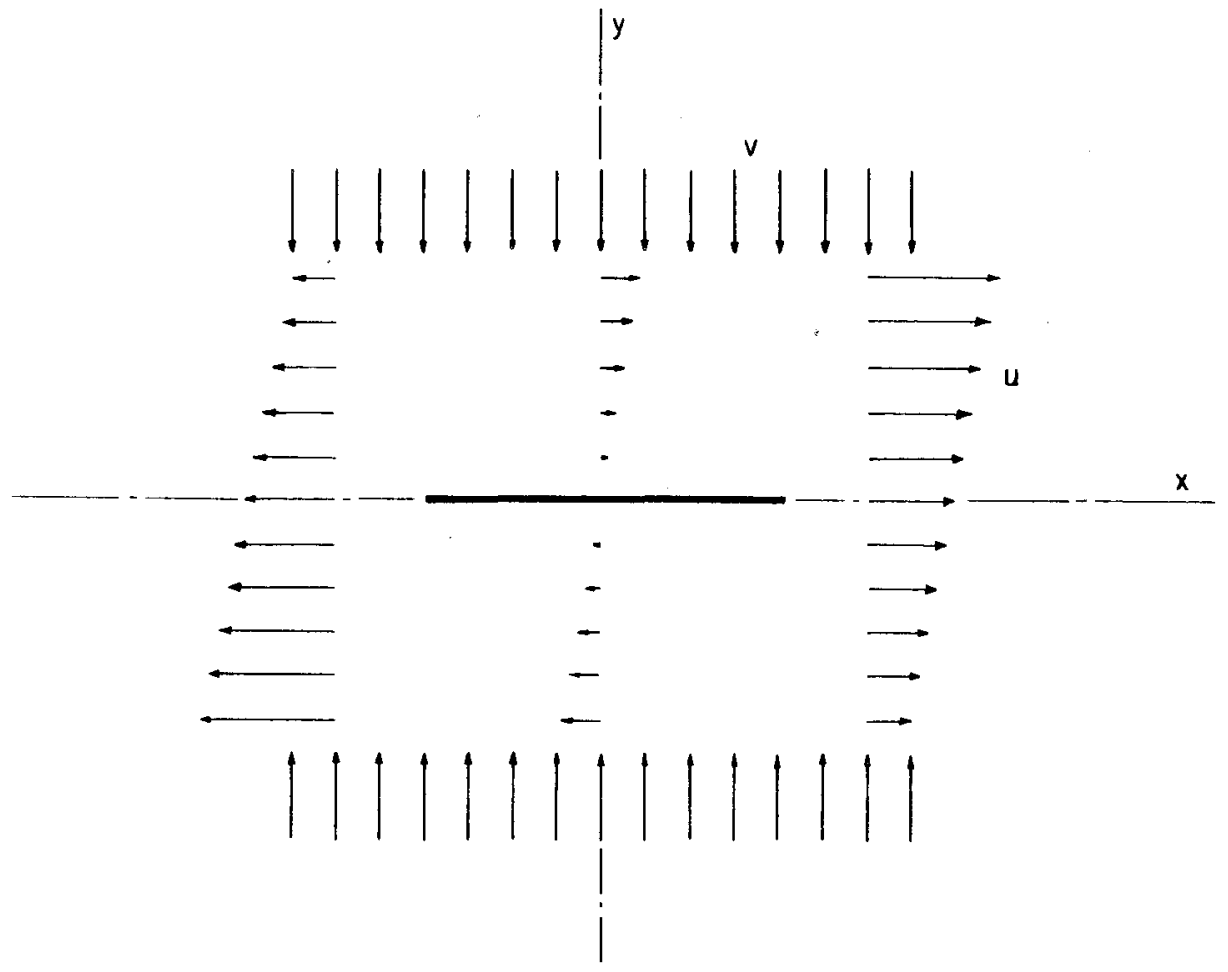

Fig. 3. Velocity field observed from a flame element in its immediate neighborhood, showing combined normal strain rate and shear rate fields.

This field is indicated schematically in Figure 3. It is particularly to be noted that when the motion corresponds to a solid-body rotation, namely $v_{\theta} \sim r$, both the straining and shearing motions vanish. This situation corresponds to our earlier observation that the pattern of the interface becomes fixed in the fluid when the viscous core has grown to the radius at which the observation is made. As the motion progresses the flame element tends to align itself with the circular arc passing through its midpoint, $\psi \rightarrow 0$, and the straining motion becomes small. The shearing motion will prove to be unimportant in the flame analysis.

The actual normal strain rate components $\varepsilon_{x x}=-\varepsilon_{y y} \equiv \varepsilon$ follow from equation (9) or (10) as

$$
\varepsilon=\frac{\partial u}{\partial x}=-\frac{\partial v}{\partial y}=r \frac{\partial}{\partial r}(\sin \psi \cos \psi)
$$

Then recalling the expression for $\tan \psi$ in equation (5), it follows that

$$
\sin \psi \cos \psi=1 /\left\{2 \zeta\left(1-e^{-R / 4 \zeta}\right)-\left[2 \zeta\left(1-e^{-R / 4 \zeta}\right)\right]^{-1}\right\}
$$


Likewise from equation (1) we find that

$$
r \frac{\partial}{\partial r}\left(v_{\theta} / r\right)=2 \zeta / t\left[1-(1+R / 4 \zeta) e^{-R / 4 \zeta}\right]
$$

and hence the strain rate may be written explicitly as

$$
\varepsilon t=\frac{4 \zeta^{2}\left(1-e^{-R / 4 \zeta}\right)\left[1-(1+R / 4 \zeta) e^{-R / 4 \zeta}\right]}{1+4 \zeta^{2}\left(1-e^{-R / 4 \zeta}\right)^{2}}
$$

For values of radius and time such that both $\zeta \ll 1$ and $\zeta / R \ll 1$ we find that $\varepsilon t \cong\left(\Gamma t / \pi r^{2}\right)^{2}$ is independent of viscosity and hence correponds to the strain rate encountered in a potential vortex. For long values of time or smaller values of $r$, i.e., $\zeta \gg 1$ and $\zeta / R \gg 1$, the viscous core dominates the field,

$$
\varepsilon t \cong(1 / 2 \zeta)(R / 2)^{3} /\left[1+(R / 2)^{2}\right]
$$

and is strongly Reynolds-number dependent. In particular, for long time values, the straining motion in the core vanishes. In the range of time and radius values such that $\zeta \gg 1$ but $\zeta / R \ll 1$ we find that $\varepsilon t \cong 1$. The range of validity is larger for larger Reynolds number, as indicated in Figure 4.

The fact that, even for very large Reynolds numbers, $\varepsilon t \leqslant 1$ has considerable significance in the time-dependent diffusion-flame theory, which will be developed subsequently.



Fig. 4. Dependence of normal strain rate-time product on dimensionless radius for various values of the Reynolds number $R \equiv \Gamma / 2 \pi \nu$. 


\section{FLAME STRUCTURE IN THE VORTEX FIELD}

We assume, principally to assure clarity in the final results, that the changes in density of the reactants do not seriously alter the results that we are seeking. Consider first the time-dependent diffusion flame, with infinitely fast kinetics, which would occur if the vortex were absent. Then at $t=0$ the mass fraction of fuel in the upper half-plane is $K_{1}(y, 0)=1$ and similarly the mass fraction of oxidizer in the lower half-plane is $K_{2}(y, 0)=1$. When the stoichiometry of the reaction is such that equal masses of each reactant are consumed at the infinitely thin reaction zone located on the horizontal axis, the reactant mass fractions satisfy identical diffusion equations which, together with the requirement that both reactants vanish on the axis, determine the reactant distributions as

$$
K_{1}=\operatorname{erf}(y / \sqrt{4 D t}) \quad \text { and } \quad K_{2}=-\operatorname{erf}(y / \sqrt{4 v t})
$$

The volume consumption rate of fuel $K_{1}$ is then, in units of volume per unit time and per unit area of the interface,

$$
D\left(\partial K_{1} / \partial y\right)(0, t)=\sqrt{D / \pi t}
$$

and the consumption rate of oxidizer is identical. The reactant consumption decreases with time as $(D / t)^{1 / 2}$ while the thickness of the diffusion zone is growing at a rate of approximately $(D t)^{1 / 2}$.

Now we employ the same ideas in performing a local analysis of the flame structure when the vortex field is present. The only difference is that the local-flame structure in the presence of the vortex must account for the velocity field in the neighborhood of the flame. These velocities are given by equations (6) and (7) in the $x-y$ coordinate systems situated at the center of the element (Figures 2 and 3). This notation is consistent with that used for the plane diffusion flame because, in that instance, all flame elements were permanently located on the horizontal axis. We make the approximation here that the normal strain rate $\varepsilon$ and the shearing strain rate $\partial u / \partial y$ are nearly independent of $x$, essentially a boundary-layer approximation, and thus the flow is locally a one-dimensional, timedependent field. Proceeding in a manner analogous to that employed above, the conservation equation for the fuel mass fraction is

$$
\frac{\partial K_{1}}{\partial t}-\varepsilon y \frac{\partial K_{1}}{\partial y}=D \frac{\partial^{2} K_{1}}{\partial y^{2}}
$$

where the relation $v=-\varepsilon y$ has been used in accordance with equations (7) and (12). If we recall that we are analyzing the flame structure in the 
neighborhood of a point $r, \theta$ on the flame interface and that $x$ and $y$ represent infinitesimal displacements from this point, then it is clear that, so far as equation (17) is concerned, $\varepsilon$ is a function of time only, $\varepsilon=\varepsilon(t)$. Furthermore it appears that, because of the independence of $x$ locally, the shearing-strain-rate term plays no part in the local flame structure.

The statement of the problem is simplified if we note that a material point, originally at $y=\bar{y}$, is transported with a velocity $v=-\varepsilon t$ and hence arrives at the location $y=\bar{y} \exp \left(-\int_{0}^{t} \varepsilon d t_{1}\right)$ after a time $t$ has elapsed. Writing equation (17) in terms of this "material" coordinate $\bar{y}$ then yields

$$
\frac{\partial K_{1}}{\partial t}=\exp \left(2 \int_{0}^{t} \varepsilon d t_{1}\right)\left(D \frac{\partial^{2} K_{1}}{\partial \bar{y}^{2}}\right)
$$

which may be viewed as a diffusion equation for a medium with timedependent properties. Then by transforming our time scale according to

$$
d \tau=\exp \left(2 \int_{0}^{t} \varepsilon d t_{1}\right) d t
$$

equation (18) takes on the familiar form

$$
\frac{\partial K_{1}}{\partial \tau}=D \frac{\partial^{2} K_{1}}{\partial \bar{y}^{2}}
$$

which may be treated in the manner of the plane flame analyzed previously. An identical argument holds for the mass fraction $K_{2}$ at negative values of $y$. The distribution of fuel mass fraction is thus

$$
K_{1}=\operatorname{erf}\left[\bar{y} /(4 D \tau)^{1 / 2}\right]
$$

and the fuel consumption rate is

$$
D \frac{\partial K_{1}}{\partial y}(0, t)=(D / \pi t)^{1 / 2} \exp \left(\int_{0}^{t} \varepsilon d t_{1}\right)
$$

The strain rate $\varepsilon$, in equation (15), depends upon the variables $r$ and $t$ but the integral in equation (22) is carried out at a fixed radius in the vortex structure. Thus we may write, using equation (15),

$$
\int_{0}^{t} \varepsilon d t_{1}=\int_{0}^{\zeta} f(\zeta ; R) d \zeta
$$

where

$$
f(\zeta ; R)=\varepsilon t / \zeta
$$


Now the transformed time scale $\tau$, which also appears in the expression for the fuel-consumption rate, is obtained by integrating equation (19). If we call

$$
\exp \left\{\int_{0}^{t} \varepsilon d t_{1}\right\}=\exp \left\{\int_{0}^{\zeta} f\left(\zeta_{1} ; R\right) d \zeta_{1}\right\} \equiv F(\zeta ; R)
$$

then the transformed time may be written in the form

$$
\Gamma \tau / 2 \pi r^{2}=\int_{0}^{\zeta} F^{2}\left(\zeta_{1} ; R\right) d \zeta_{1} \equiv G^{2}(\zeta ; R)
$$

and, from equation (22), the volume rate of fuel consumption per unit area of flame surface is

$$
\left[(D \Gamma)^{1 / 2} /(\sqrt{2} \pi r)\right] F(\zeta ; R) / G(\zeta ; R)
$$

This expression is valid at all points on the flame in the vortex field under the assumptions that the variations of the strain components along the flame are small over distances comparable with the flame-zone thickness and that adjacent flame surfaces do not interfere.

Eventually, however, adjacent flame sheets will interact in the sense that the diffusion-zone thickness about the flames becomes comparable with the spacing of the wound-up flame surfaces. As the overlap of the diffusion zones depletes the concentration of reactant between adjacent flame surfaces, the reactant consumption rate will decrease and the two flame sheets eventually annihilate each other. The nature of the end process may be analyzed by considering a strip of reactant (say, the fuel component), which is being strained along its length at a rate $\varepsilon$. If the two edges of the fuel strip are at the points $y= \pm y_{0}$ at $t=0$, the time the diffusion flames start, then the straining motion itself moves these flame fronts to the instantaneous position $\pm y_{0} \exp \left\{-\int_{0}^{t} \varepsilon d t_{1}\right\}$, hence decreasing the width of the fuel strip. Then the transformations used for the single strained flame are appropriate; the mass-fixed coordinate

$$
\bar{y}=y \exp \left(-\int_{0}^{r} \varepsilon d t_{1}\right)
$$

and the effective time

$$
\tau=\int_{0}^{t}\left[\exp \left(2 \int_{0}^{t_{2}} \varepsilon d t_{1}\right)\right] d t_{2}
$$


reduce the problem to a conventional diffusion equation. It is not difficult to show that, within the fuel strip, the mass fraction of fuel is

$$
K_{1}=-1-\operatorname{erf}\left[\left(\bar{y}-y_{0}\right) /(4 D \tau)^{1 / 2}\right]+\operatorname{erf}\left[\left(\bar{y}+y_{0}\right) /(4 D \tau)^{1 / 2}\right]
$$

At either flame sheet $K_{1}=0$, a condition which determines the positions $\bar{y}_{\mathrm{f}}$ and $-\bar{y}_{f}$ of the flame sheets. The volume consumption rate of fuel by each of the flame sheets is

$$
\sqrt{\frac{D}{\pi \tau}}\left\{\exp \left[-\left(\frac{\bar{y}_{\mathrm{f}}-y_{0}}{(4 D \tau)^{1 / 2}}\right)^{2}\right]-\exp \left[-\left(\frac{\bar{y}_{\mathrm{f}}+y_{0}}{(4 D \tau)^{1 / 2}}\right)^{2}\right]\right\}
$$

which vanishes as the flames collapse, i.e., as $\bar{y}_{f} \rightarrow 0$. It is a straightforward calculation to show that the two flames behave independently, except for a brief final period $\cong 1 / \varepsilon$ when the combustion of the fuel strip is abruptly completed.

For our purpose here, this abrupt termination of the burning process may be used to advantage. We shall adopt the approximation that the reactant consumption of the flames proceeds as if they were independent until the fuel supply is exhausted, at which time the flames bounding the fuel (or oxidizer) strip terminate instantaneously.

\section{INCREASE OF REACTANT CONSUMPTION CAUSED BY THE VORTEX FIELD}

The length $d s$ of the flame element that extends from a radius $r$ to $r+d r$ is

$$
\begin{aligned}
d s & =\left[1+\left(r \frac{\partial \theta}{\partial r}\right)^{2}\right]^{1 / 2} d r \\
& =\left\{1+\left[2 \zeta\left(1-e^{-R / 4 \zeta}\right)\right]^{2}\right\}^{1 / 2}
\end{aligned}
$$

where equation (2) has been used to obtain the final expression. The rate at which fuel is being consumed by the two flames that bound this annulus is then

$$
(1 / \pi)(2 D \Gamma)^{1 / 2}(F / G)\left\{1+\left[2 \zeta\left(1-e^{-R / 4 \zeta}\right)\right]^{2}\right\}^{1 / 2} d r / r
$$

Corresponding to our earlier discussion we shall make the quite accurate approximation that the reactant consumption rate is described by equation 
(33) until the fuel in the annulus $(r, d r)$ has been consumed and, at that point, the local consumption rate vanishes. In other words, the condition for burnout of the annulus $(r, d r)$ is that the integral of the volume rate of fuel consumption, equation (33), over time shall equal the volume of fuel within the annulus, namely $\pi r d r$. Thus the time $t_{*}$ at which the fuel and oxidizer in the annulus $(r, d r)$ are consumed is determined by the integral equation

$$
(1 / \pi)(2 D \Gamma)^{1 / 2} d r / r \int_{0}^{t_{*}}(F / G)\left\{1+\left[2 \zeta\left(1-e^{-R / 4 \zeta}\right)\right]^{2}\right\}^{1 / 2} d t=\pi r d r
$$

and because $r$ is constant during this integration we may write

$$
(2 / \pi)(2 D / \Gamma)^{1 / 2} \int_{0}^{\zeta_{*}}(F / G)\left\{1+\left[2 \zeta\left(1-e^{-R / 4 \zeta}\right)\right]^{2}\right\}^{1 / 2} d \zeta=1
$$

The value of $\zeta_{*}$ determined by this integral may be interpreted as giving the radius $r_{*}$ at which the reactants have been consumed at any time $t$. In fact it is clear now that the structure of the vortex combustion is divided into two distinct parts: (i) an inner core in which the combustion has been completed and (ii) an outer flame structure in which the two arms of the flame sheet may be described locally by the nonsteady diffusion-flame theory of Section 3 . The circle which separates these two zones has a radius $r_{*}=\left(\Gamma / 2 \pi \zeta_{*}\right)^{1 / 2} t^{1 / 2}$.

It is a consequence of the fact that the radius of the core increases as $t^{1 / 2}$ that the rate at which combustion products are accumulated within the core is a constant, independent of time. This follows because the volume of fuel consumed is just half of the volume swept out by the increasing core radius and consequently the volume rate of fuel consumption in the core is $\frac{1}{2} d\left(\pi r_{*}^{2}\right) / d t=4 \Gamma / \zeta_{*}$. Referring to equation (34) it is clear that $\zeta_{*}$ depends upon the Reynolds number $\Gamma / 2 \pi \nu$ and the ratio $D / \Gamma$; thus both the radius of the reacted core and the reactant consumption rate will vary with these quantities. It is clear that this dependence can equally well be expressed in terms of the Reynolds number and $\nu / D$, the Schmidt number.

It is a matter of some calculation to determine $\zeta_{*}$ as a function of Reynolds number and Schmidt number, but another representation proves to be more convenient and physically more penetrating. When the Reynolds number is large and $\zeta_{*}=\Gamma t / 2 \pi r_{*}^{2}$ is large, it transpires that (i) the winding of the flame sheet is quite tight and (ii) the core of combustion products grows more rapidly than the viscous core. In this limit the behavior of the field is essentially inviscid and equation (34) may be examined by elementary asymptotic methods. Consider $R / 4 \zeta_{*} \gg 1$ and $\zeta_{*} \gg 1$; then it is easily shown that $F(\zeta ; R) \rightarrow\left(1+4 \zeta^{2}\right)^{1 / 2}$ and 
$G^{2}(\zeta ; R) \rightarrow \zeta\left(1+4 \zeta^{2} / 3\right)$, with the consequence that the integral relation (34) becomes

$$
(2 / \pi)(2 D / \Gamma)^{1 / 2} \int_{0}^{\zeta_{*}} \frac{1+4 \zeta^{2}}{\left(1+4 \zeta^{2} / 3\right)^{1 / 2}} \zeta^{-1 / 2} d \zeta \cong 1
$$

The integral behaves as $\zeta^{-1 / 2}$ for small $\zeta$ and as $2 \sqrt{3} \zeta^{1 / 2}$ for large $\zeta$ and it is convenient to rewrite equation (35) as

$$
(2 / \pi)(2 D / \Gamma)^{1 / 2}\left[\frac{4}{\sqrt{3}} \zeta_{*}^{3 / 2}+I(\infty)-2 \sqrt{3} \int_{\zeta_{*}}^{\infty}\left(\frac{1+1 / 4 \zeta^{2}}{\left(1+3 / 4 \zeta^{2}\right)^{1 / 2}}-1\right) \zeta^{-1 / 2} d \zeta\right]
$$

where

$$
I(\infty)=2 \sqrt{3} \int_{0}^{\infty}\left[\frac{1+4 \zeta^{2}}{\left(1+4 \zeta^{2} / 3\right)^{1 / 2}}-1\right] \zeta^{1 / 2} d \zeta=0.1994
$$

The dominant term $\zeta_{*}^{3 / 2}$ yields the lowest-order approximation,

$$
\zeta_{*}(D / \Gamma)^{1 / 3} \cong(\sqrt{3 / 2} \pi / e)^{2 / 3}
$$

The dimensionless group

$$
\zeta_{*}(D / \Gamma)^{1 / 3}=\Gamma^{2 / 3} D^{1 / 3} t / 2 \pi r_{*}^{2}
$$

is of a form common to diffusion problems except that it contains an unusual composite transport property $\Gamma^{2 / 3} D^{1 / 3}$. If the core were growing by a molecular-diffusion process we should expect its area to increase as $D t$ but the growth rate for our problem, $\sim(\Gamma / D)^{2 / 3} D t$, is enormously faster. Physically this result arises because the full diffusion potential occurs across each narrow strip of the strongly wound-up pattern.

Based upon this observation it is appropriate to use the dependent variable $\Gamma^{2 / 3} D^{1 / 3} t / 2 \pi r_{*}^{2}$ in preference to $\zeta_{*}$ because it is, in the lowest approximation, independent of both Reynolds and Schmidt numbers. Then a conventional asymptotic evaluation of equation (35) for large $\zeta_{*}$ may be used to express

$$
2 \pi r_{*}^{2} / \Gamma^{2 / 3} D^{1 / 3} t \cong(8 \sqrt{2 / 3} / \pi)^{2 / 3}+O(D / \Gamma)^{1 / 2}
$$

Returning now to the complete formulation given by equation (34), it is, not difficult to evaluate $\zeta_{*}$ numerically and thereby determine $2 \pi r_{*}^{2} / \Gamma^{2 / 3} D^{1 / 3} t$ as a function of Reynolds number and Schmidt number. Figure 5 shows such a result for $\nu / D=1.0$ and shows the essential 


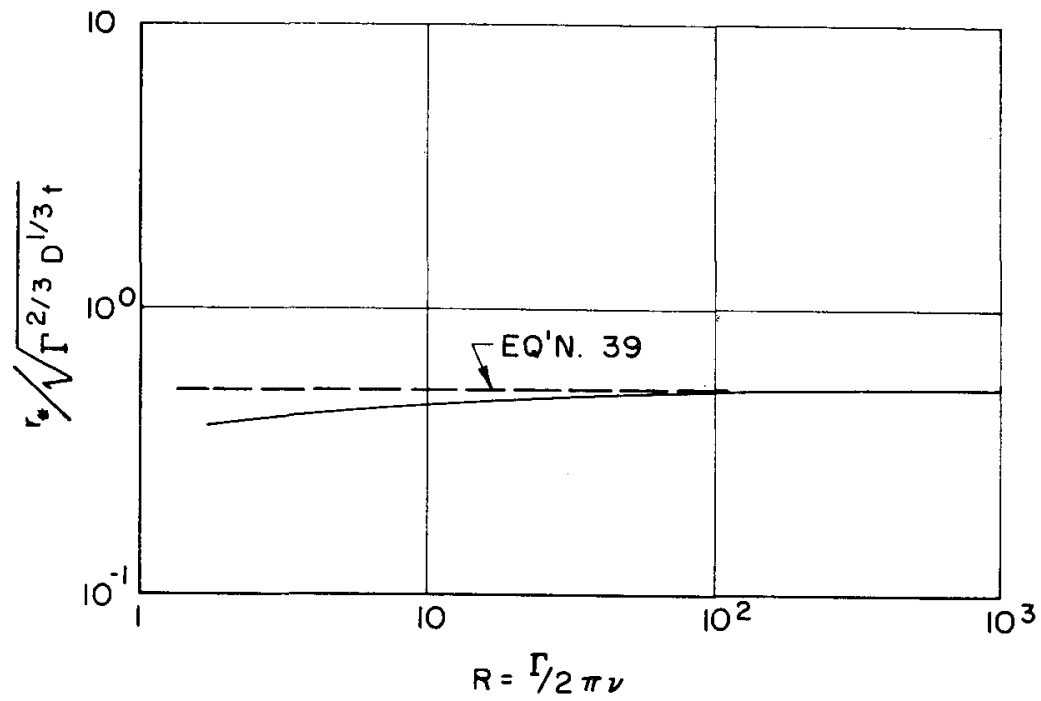

Fig. 5. Dimensionless radius of reacted core $r_{*} /\left(\Gamma^{2 / 3} D^{1 / 3} t\right)^{1 / 2}$ as a function of Reynolds number $R \equiv \Gamma / 2 \pi \nu$ for Schmidt number $(\nu / D) \stackrel{*}{=} 1.0$.

independence of Reynolds number, certainly for $R>50$. The asymptotic value calculated from equation (39) is also shown and clearly provides a very satisfactory representation over most of the range.

To account for the reactant-consumption rate we again proceed from equation (32), which gives the fuel-consumption rate for an isolated flame element situated in an annulus of radius $r$ and thickness $d r$. It is convenient to compute the augmentation of reactant-consumption rate caused by the vortex-flow field and consequently we subtract the consumption rate that would be experienced by an element of length $d r$ if it remained on the horizontal axis in its original position, in the absence of the vortex. This reactant consumption rate for the undistorted flame element is given by equation (16).

The net increase in fuel-consumption rate within $d r$, accounting for both branches of the flame, is

$$
(1 / \pi)(2 D \Gamma)^{1 / 2}(F / G)\left\{1+\left[2 \zeta\left(1-e^{-R / 4 \zeta}\right)\right]^{2}\right\}^{1 / 2} \frac{d r}{r}-2(D / \pi t)^{1 / 2} d r
$$

The net increase in fuel-consumption rate, associated with the vortexinduced motion, over the entire field follows by integrating this expression from $r_{*}$ to $\infty$, i.e., from $\zeta=0$ to $\zeta=\zeta_{*}$. The result is

$$
(1 / \pi)(D \Gamma / 2)^{1 / 2} \int_{0}^{\zeta_{*}}\left[(F / G)\left\{1+\left[2 \zeta\left(1-e^{-R / 4 \zeta}\right)\right]^{2}\right\}^{1 / 2}-\zeta^{-1 / 2}\right] d \zeta / \zeta
$$


Again, as in our evaluation of the core radius $r_{*}$, it is instructive to find the ideal limit of large Reynolds number where the conditions $\zeta_{*} \gg 1$ and $R / 4 \zeta_{*} \gg 1$ are satisfied. Utilizing some previous results, the expression for the augmented consumption rate is

$$
\cong(1 / \pi)(D \Gamma / 2)^{1 / 2} \int_{0}^{\zeta_{*}}\left(\frac{1+4 \zeta^{2}}{\left(1+4 \zeta^{2} / 3\right)^{1 / 2}}-1\right) \zeta^{-3 / 2} d \zeta
$$

For asymptotic evaluation rewrite this as

$$
(1 / \pi)(D \Gamma / 2)^{1 / 2}\left\{4 \sqrt{3} \zeta_{*}^{1 / 2}+J(\infty)-\int_{\zeta_{*}}^{\infty}\left[\frac{1+4 \zeta^{2}}{\left(1+4 \zeta^{2} / 3\right)^{1 / 2}}-2 \sqrt{3} \zeta-1\right] \zeta^{-3 / 2} d \zeta\right\}
$$

where

$$
\begin{aligned}
J(\infty) & =\int_{0}^{\infty}\left[\frac{1+4 \zeta^{2}}{\left(1+4 \zeta^{2} / 3\right)^{1 / 2}}-2 \sqrt{3} \zeta-1\right] \zeta^{-3 / 2} d \zeta \\
& =-6.4543
\end{aligned}
$$

The integral in equation (43) may be evaluated asymptotically for large $\zeta_{*}$ and, regrouping the variables in the manner found convenient for coreradius evaluation, the augmented consumption rate is

$$
\Gamma^{2 / 3} D^{1 / 3}\left\{\frac{4}{\pi} \sqrt{\frac{3}{2}}\left(\frac{\Gamma^{2 / 3} D^{1 / 3} t}{2 \pi r_{*}^{2}}\right)^{1 / 2}+\frac{1}{\sqrt{2} \pi} J(\infty)\left(\frac{D}{\Gamma}\right)^{1 / 6}+O\left(\frac{D}{\Gamma}\right)^{1 / 2}\right\}
$$

Because the second term in this expression is decreased only by the factor $(D / \Gamma)^{1 / 6}=(2 \pi)^{-1 / 6}(\mathrm{Sc} \cdot R)^{-1 / 6}$, it shall be included although higher-order terms may be neglected.

We conclude from equation (45) that the augmented reactant consumption rate scales as $\Gamma^{2 / 3} D^{1 / 3}$, the composite transport property we found earlier. Inserting known values of $\Gamma^{2 / 3} D^{1 / 3} t / 2 \pi r_{*}^{2}$ and $J(\infty)$, equation (45) for the augmented consumption rate becomes

$$
\cong \Gamma^{2 / 3} D^{1 / 3}\left[1.2327-1.0694(\mathrm{Sc} \cdot R)^{-1 / 6}+O(D / \Gamma)^{1 / 2}\right]
$$

Note particularly that writing $D / \Gamma \sim(\mathrm{Sc} \cdot R)^{-1}$ has a certain physical convenience but does not imply the involvement of viscosity.

Now it is a simple matter to compute from the complete representation, equation (41), the quantity $\Gamma^{-2 / 3} D^{-1 / 3}$ (augmented consumption rate) as suggested by our considerations for high Reynolds numbers. Such 


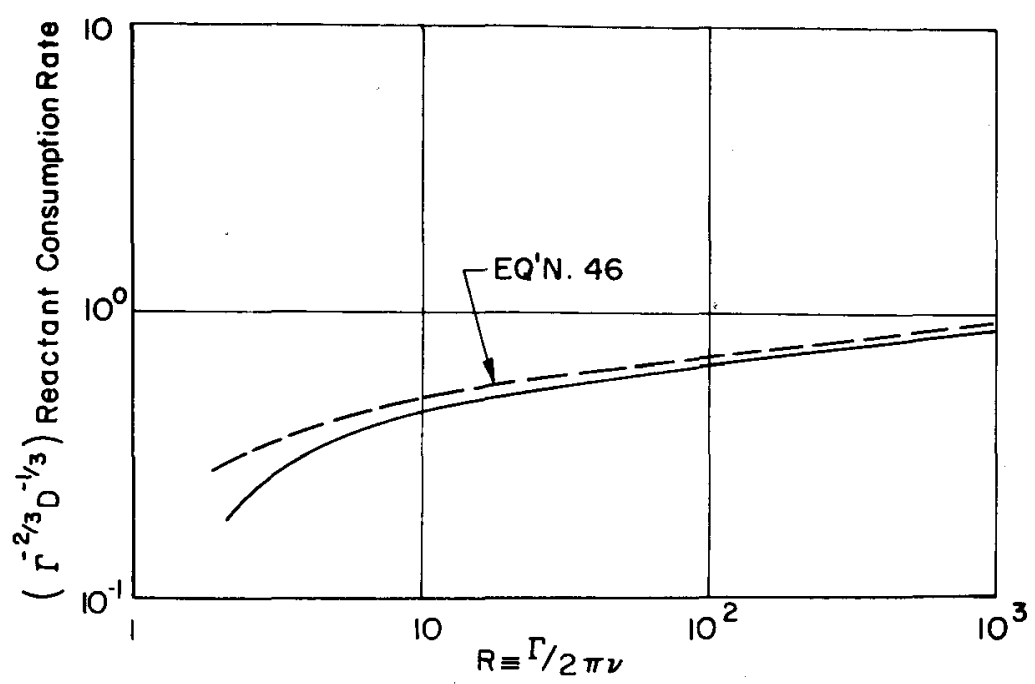

Fig. 6. Reactant consumption rate as a function of Reynolds number $R \equiv \Gamma / 2 \pi \nu$ for Schmidt number $(\nu / D)=1.0$.

calculations are given in Figure 6 as a function of Reynolds number for $\nu / D=1.0$. The scaling with the composite transport coefficient $\Gamma^{2 / 3} D^{1 / 3}$ is generally quite adequate. The asymptotic formula given by equation (46) is surprisingly accurate and the significance of the $R^{-1 / 6}$ term is particularly to be noted. Again, it is to be emphasized that the augmentation in combustion rate caused by the vortex field is independent of time, in spite of the fact that the velocity field, flame shape, and core size are time-dependent.

Because these results are significant not only in the gas-phase combustion process but also for reactions in liquids, it is of interest to explore the influence of the ratio of kinematic viscosity to diffusion coefficients, or Schmidt number. This ratio is the significant quantity that changes drastically between the two cases. The Schmidt number is also of interest because it is frequently convenient to explore combustion processes in gases by examining their analogues in liquids. Figure 7 shows the effect of $\nu / D$ upon the reactant consumption rates; the Reynolds number is constant at a value $R=1000$ and the $\nu / D$ ratio ranges from $10^{-2}$ to $10^{3}$. The computed values of the dimensionless core size are essentially independent of the Schmidt number and are indistinguishable from the asymptotic formula, equation (39), over the same range of Schmidt numbers. 


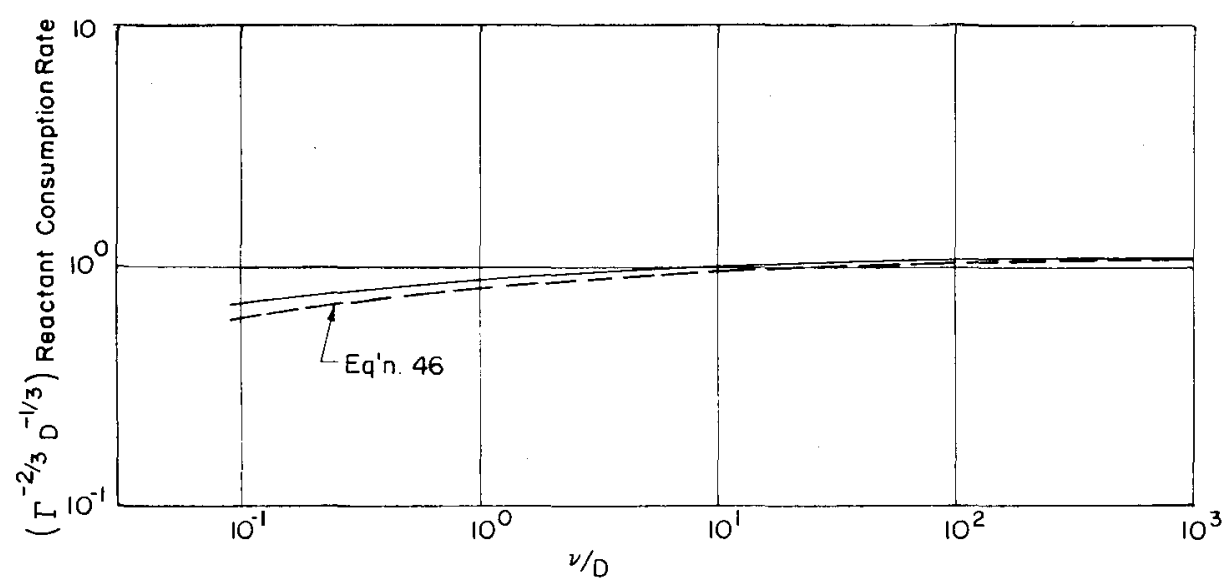

Fig. 7. Reactant consumption rates as function of $\nu / D=\mathrm{Sc}$, Reynolds number $\Gamma / 2 \pi \nu=10^{3}$.

\section{CONCLUDING REMARKS}

The foregoing results have a relatively simple asymptotic behavior because most of the diffusion and combustion process lies sufficiently outside the viscous core that the velocity field may be considered a potential vortex. Because of this it is possible to arrive at these asymptotic forms in a manner physically more instructive than formal analysis.

Recall that for a potential vortex the length of flame $d s$ that spans a radial element $d r$ is given by equation (4), so that when the flame makes a complete circuit of the origin, $d s=2 \pi r$ and the distance between these neighboring flame sheets is $d r=\left(2 \pi r^{2} / \Gamma t\right)(2 \pi r)$. Now the thickness of the diffusion layer accompanying this flame sheet grows as $(D / \varepsilon)^{1 / 2} \cong(D t)^{1 / 2}$, since in this range of Reynolds number (cf Figure 4) $\varepsilon t \cong 1$. The flame sheets interact and consume the intervening reactant when $(D t)^{1 / 2} \cong d r$. From the above relations $(D t)^{1 / 2} \sim r_{*}^{3} / \Gamma t$, from which it follows that

$$
r_{*} /\left(\Gamma^{2 / 3} D^{1 / 3} t\right)^{1 / 2}=\text { const }
$$

Therefore the proposed similarity-law radius of the reaction product core, equation (39), follows immediately from physical reasoning.

Likewise the reactant consumption rate may be deduced by noting that the rate of accumulation of products in the core is proportional to

$$
\frac{d}{d t}\left(\pi r_{*}^{2}\right) \sim \frac{d}{d t}\left(\Gamma^{2 / 3} D^{1 / 3} t\right) \sim \Gamma^{2 / 3} D^{1 / 3}
$$


Thus the similarity law for the time-invariant reactant-consumption rate, equation (46), follows from the same approximate physical picture. The lower limit of validity for these relations must depend upon $\Gamma^{2 / 3} D^{1 / 3}$ and hence it is appropriate to state it in terms of $R \cdot \mathrm{Sc}^{1 / 2}$. From the calculations it appears that the combustion similarity laws are accurately valid for $R \cdot \mathrm{Sc}^{-1 / 2} \geqslant 50$.

A final word may be added regarding the effects of a change in fluid species, especially between gases and liquids. If we have species designated 1 and 2, the similarity law suggests that

$$
\frac{r_{* 2}}{r_{* 1}}=\frac{\Gamma_{2}^{1 / 3} D_{2}^{1 / 6}}{\Gamma_{1}^{1 / 3} D_{1}^{1 / 3}}=\left(\frac{\nu_{2}}{\nu_{1}}\right)^{1 / 2}\left(\frac{R_{2}}{R_{1}}\right)^{1 / 3}\left(\frac{\mathrm{Sc}_{1}}{\mathrm{Sc}_{2}}\right)^{1 / 6}
$$

and that the reactant consumption rates scale as

$$
\frac{\Gamma_{2}^{2 / 3} D_{2}^{1 / 3}}{\Gamma_{1}^{2 / 3} D_{1}^{1 / 3}}=\left(\frac{\nu_{2}}{\nu_{1}}\right)\left(\frac{R_{2}}{R_{1}}\right)^{2 / 3}\left(\frac{\mathrm{Sc}_{1}}{\mathrm{Sc}_{2}}\right)^{1 / 3}
$$

Comparing, for example, a liquid (2) with a gas (1) for the same Reynolds number, the reacted-core radii would be in the ratio of about $1 / 10$ while the production of reactant products is in the ratio of about $1 / 100$.

ACKNOWLEDGMENT. This work was supported, in part, by grant AFOSR$80-0265$ and by NASA grant NAG 3-70.

\section{REFERENCES}

1. G. F. Carrier, F. E. Fendell, and F. E. Marble, Effect of strain rate on diffusion flames, SIAM J. Appl. Math. 28, 453 (1975).

2. D. B. Spalding, Theory of mixing and chemical reaction in the opposed-jet diffusion flame, ARS J. 31, 763 (1961).

3. F. E. Marble and J. E. Broadwell, The Coherent Flame Model for Turbulent Chemical Reactions, Project Squid Technical Report TRW-9-PU (January, 1977).

4. Sir H. Lamb, Hydrodynamics, sixth edn., p. 592, Cambridge University Press (1932).

5. H. C. Hottel, Burning in laminar and turbulent fuel jets, in: Fourth Symp. (Int.) on Combust., p. 97, William \& Wilkins Co., Baltimore (1952). 